\title{
Improved ground-state electronic structure and optical dielectric constants with a semilocal exchange functional
}

\author{
Vojtech Vlcek, Gerd Steinle-Neumann, Linn Leppert, Rickard Armiento and Stephan \\ Kuemmel
}

\section{Linköping University Post Print}

\section{Tweet}

N.B.: When citing this work, cite the original article.

Original Publication:

Vojtech Vlcek, Gerd Steinle-Neumann, Linn Leppert, Rickard Armiento and Stephan Kuemmel, Improved ground-state electronic structure and optical dielectric constants with a semilocal exchange functional, 2015, Physical Review B. Condensed Matter and Materials Physics, (91), 3, 035107.

http://dx.doi.org/10.1103/PhysRevB.91.035107

Copyright: American Physical Society http://www.aps.org/

Postprint available at: Linköping University Electronic Press http://urn.kb.se/resolve?urn=urn:nbn:se:liu:diva-114585 


\title{
Improved ground-state electronic structure and optical dielectric constants with a semilocal exchange functional
}

\author{
Vojtěch Vlček, ${ }^{1, *}$ Gerd Steinle-Neumann, ${ }^{1}$ Linn Leppert, ${ }^{2}$ Rickard Armiento, ${ }^{3}$ and Stephan Kümmel ${ }^{2}$ \\ ${ }^{1}$ Bayerisches Geoinstitut, Universität Bayreuth, D-95440 Bayreuth, Germany \\ ${ }^{2}$ Theoretische Physik IV, Universität Bayreuth, D-95440 Bayreuth, Germany \\ ${ }^{3}$ Department of Physics, Chemistry and Biology (IFM), Linköping University, SE-58183 Linköping, Sweden
}

(Received 2 June 2014; revised manuscript received 14 October 2014; published 6 January 2015)

\begin{abstract}
A recently published generalized gradient approximation functional within density functional theory (DFT) has shown, in a few paradigm tests, an improved KS orbital description over standard (semi)local approximations. The characteristic feature of this functional is an enhancement factor that diverges like $s \ln (s)$ for large reduced density gradients $s$ which leads to unusual properties. We explore the improved orbital description of this functional more thoroughly by computing the electronic band structure, band gaps, and the optical dielectric constants in semiconductors, Mott insulators, and ionic crystals. Compared to standard semilocal functionals, we observe improvement in both the band gaps and the optical dielectric constants. In particular, the results are similar to those obtained with orbital functionals or by perturbation theory methods in that it opens band gaps in systems described as metallic by standard (semi)local density functionals, e.g., Ge, $\alpha$-Sn, and CdO.
\end{abstract}

DOI: 10.1103/PhysRevB.91.035107

PACS number(s): 71.20.-b, 71.15.Mb, 78.20.Ci

\section{INTRODUCTION}

The Kohn-Sham (KS) [1] approach to density functional theory (DFT) [2] has become the standard tool for electronic structure computations in solid state physics and materials science. It owes its success to the generally favorable compromise between accuracy and computational efficiency offered by (semi)local approximations to the exchange-correlation (xc) functional. However, (semi)local approximations are far from flawless. In particular, for KS single particle states that are supposed to be very localized, (semi)local functionals frequently give orbitals that are too delocalized and too high in energy. This shortcoming is a widely recognized problem, which is often considered a consequence of various aspects of the self-interaction error, arguably the major source of error in DFT calculations (see, e.g., Ref. [3] for an expanded discussion).

There is a large library of methods that aim for an improved description of localized states, and the development of new approaches is an active field of research. Higher-order or beyond DFT methods, such as hybrid functionals [4] (e.g., Ref. [5]), exact exchange with the optimized effective potential (xOEP) [6,7] and the GW method [8] represent a viable solution, which however come at vastly increased computational expense. While the quality of the orbital description in $\mathrm{KS}$-DFT is an inherently difficult topic, as the KS states do not directly represent electron quasiparticle states in the usual sense, a comparison with the above mentioned higher-order methods shows that the (semi)local functionals tend to overdelocalize the KS orbitals in general. Other methods aiming at remedying this problem require nonfundamental speciesand environment-dependent parameters (DFT $+U)$ [9], or abandon the variational KS-DFT framework, e.g., model potentials such as the Becke-Johnson potential [10] and its modifications, e.g., by Tran and Blaha (TB-mBJ) [11] or others [12]. However, some of us recently developed a

\footnotetext{
*vojtech.vlcek@uni-bayreuth.de
}

standard semilocal generalized gradient approximation (GGA) functional that improves the KS orbital description, while it still remains fully within the KS-DFT framework, the Armiento-Kümmel functional (AK13) [13]. Unfortunately, this functional comes with its own tradeoffs, e.g., a lower accuracy of the total energy, clearly demonstrated in the supplementary material of Ref. [13] and further commented on by Ref. [14]. It is nevertheless encouraging that it is at all possible for a semilocal functional to make a significant qualitative improvement of the orbital description compared to results of other (semi)local functionals. The purpose of the present paper is to more closely investigate the nature of this improvement in the context of crystalline solids.

From both the pragmatic and the fundamental perspective it is highly desirable to have xc functionals which qualitatively capture the important physics of a system. As discussed above, one such aspect is the improved localization of the KS orbitals seen with higher-order methods. Physical properties calculated from properly localized KS orbitals often compare favorably with experimental results (independently of how one justifies this practice, cf. Secs. II and VII). In this paper we study the Kohn-Sham band structure and optical dielectric constants for a variety of solids, including Mott insulators, semiconductors, and insulators. Our focus is not to examine the accuracy with which the various methods reproduce experimental results but rather the extent to which the AK13 functional shares the qualitative overall improvement of higher-order methods over standard (semi)local functionals. As explained, such improvements can be expected to bring the results of properties derived from the KS orbitals closer to the experimental values.

Our paper is organized as follows: Section II gives a more in-depth discussion of the interpretation of the KS orbitals and the KS band gap; in Sec. III we summarize the essentials of the construction of the AK13 functional; Sec. IV covers the computational details of our study; in Secs. V and VI we present and discuss the results for band gaps and optical dielectric constants, respectively; Sec. VII provides an outlook and a summary of the results obtained here. 


\section{KS ORBITALS AND THE KS BAND GAP: RELATION TO PHYSICAL PROPERTIES}

A self-consistent KS calculation gives as its results a total ground-state energy $E$ and a set of eigenvalues $\left\{\varepsilon_{i}\right\}$. The energy $E$ is the central observable of ground-state DFT, and there is no doubt about its physical meaning. Contrary to $E$, it is not guaranteed that the $\left\{\varepsilon_{i}\right\}$ have a well defined physical meaning; even for the exact xc functional only the highest occupied one [15] can be interpreted as the first ionization potential $I$ for finite systems. In early DFT the eigenvalues were thus denied any meaning at all [16]. However, experience has shown that the KS band structure, i.e., the $\left\{\varepsilon_{i}\right\}$ obtained in periodic-boundary condition calculations for crystals, is extremely useful for practical purposes [17,18], despite the eigenvalues and the KS potential already having an unknown absolute energy offset relative to the vacuum level. We are thus restricted to the interpretation of eigenvalue differences: ${ }^{1}$ By now it has been firmly established that relative energies of occupied KS eigenstates can accurately approximate ionization potentials $I$ [19], and, e.g., photoemission experiments have even confirmed the physical interpretability of orbitals themselves [20-22].

The situation is very different for the unoccupied eigenvalues, which cannot be associated with electron affinities $A$ or inverse photoemission spectra energies. This is even true for the exact xc functional, due to the derivative discontinuity $\Delta_{\mathrm{xc}}$ [23]. Because of $\Delta_{\mathrm{xc}}$, the relative energies between the occupied and unoccupied KS orbitals do not correspond to quasiparticle energy differences. As a result of this the KS gap, defined as the energy difference between the lowest unoccupied $\left(\varepsilon_{L}\right)$ and highest occupied $\left(\varepsilon_{H}\right)$ KS eigenvalue $E_{g}^{\mathrm{KS}}=\varepsilon_{L}-\varepsilon_{H}$, is in general not equal to the fundamental gap [24-27], defined as $E_{g}=I-A$. The two gaps differ by $\Delta_{\text {xc }}$, i.e.,

$$
E_{g}=E_{g}^{\mathrm{KS}}+\Delta_{\mathrm{xc}} .
$$

Despite the fact that $E_{g}^{\mathrm{KS}}$ does not represent the fundamental gap $E_{g}$, KS eigenvalues are a very important result of electronic structure calculations for at least three reasons:

(i) Regardless of the fact that the $E_{g}^{\mathrm{KS}}$ from (semi)local functionals typically underestimates the experimental gap noticeably, experience for periodic systems shows that the shape and general features of KS bands are often physically meaningful and have pragmatically been used with great success $[18,28,29]$.

(ii) For finite systems, $E_{g}^{\mathrm{KS}}$ represents the energy of an excitation which can be described (approximately) as an electron and hole being close to each other [30], and, as such, it cannot be interpreted as a good approximation for $E_{g}$. A better interpretation of KS eigenvalue differences is as a zero order approximation of excitation energies $[31,32]$.

\footnotetext{
${ }^{1}$ In the context of periodic solids, it is only meaningful to look at the relative energies of the KS orbitals as their absolute values cannot be related to the vacuum level. This should be clear from the observation that the work function of a real solid depends on the surface, and thus, the absolute levels are affected by regions not included in the infinite periodic model.
}

(iii) The KS band structure often serves as an input for calculating quasiparticle energies in the GW approximation $[8,33,34]$. It has also been argued that the xc potential can be interpreted as the best local approximation to the self energy in Dyson's equation [35].

As outlined in the introduction, independent of the question of interpretation of the eigenvalues, xOEP tends to improve the similarity of the KS band structure with energies obtained from higher-order and quasiparticle theory [34,36-39]. Also, hybrid functionals which include (some) generalized KS exact exchange improve band-structure prediction [40]. The magnitude of self-interaction errors [41,42] and the question of whether the KS or the generalized KS scheme is used [43] are both important for the interpretability of eigenvalues. The considerable improvement from full or partial exact exchange or self-interaction corrections, however, comes at the high computational price of having to evaluate many exchange- or Coulomb integrals. The recently developed AK13 functional spurs hopes that these deficiencies can be mitigated at the computational cost of a standard (semi)local functional.

\section{SEMILOCAL DFT WITH AN IMPROVED ORBITAL DESCRIPTION}

The AK13 is an exchange-only density functional, which was inspired by the exchange potential derived by Becke and Johnson [10], $v_{x}^{\mathrm{BJ}}(\mathbf{r})$. It can be expressed in terms of the charge density $n(\mathbf{r})$, and its KS potential for atoms approximates that of xOEP. By requiring the exchange potential to have the same asymptotic properties as $v_{x}^{\mathrm{BJ}}(\mathbf{r})$, one can derive [13] the following restriction on a corresponding GGA exchange energy functional:

$$
E_{x}=A_{x} \int n(\mathbf{r}) F(s) \mathrm{d} \mathbf{r}
$$

with

$$
F(s) \rightarrow c \cdot s \ln (s) \text { for } s \rightarrow \infty,
$$

where $A_{x}=-(3 / 4)(3 / \pi)^{1 / 3}$ in Hartree atomic units. $F(s)$ is the enhancement factor, $c$ a numerical constant, and $s$ the reduced density gradient

$$
s=\frac{|\nabla n(\mathbf{r})|}{2\left(3 \pi^{2}\right)^{1 / 3} n(\mathbf{r})} .
$$

The AK13 functional implements this requirement and a few other restrictions by the following expression for the enhancement factor

$$
F(s)=1+B_{1} s \ln (1+s)+\left(\mu_{\mathrm{GE}}-B_{1}\right) s \ln [1+\ln (1+s)],
$$

where $\mu_{\mathrm{GE}}=10 / 81$, and $B_{1}=3 / 5 \mu_{\mathrm{GE}}+8 \pi / 15$ is a constant which determines the strength of the discontinuity of the potential. All the constants in the above expression were obtained from theoretical considerations for the exchange potential outside of a finite system and semi-infinite surfaces, i.e., Eq. (5) contains no empirical parameter.

The AK13 exchange functional is a KS-DFT functional of GGA form, i.e., it is semilocal in $n(\mathbf{r})$, and thus allows computations at a cost similar to other (semi)local functionals. However, it was argued in Ref. [13] that any functional whose 
KS potential takes a nonzero asymptote outside a finite system requires some care in the definition of the zero of energy in the KS system. Specifically, to interpret the potential and KS eigenvalues as discussed in Sec. II, they must first be shifted with precisely the constant value that aligns the potential asymptote to zero, $c_{\text {shift }}$. However, this shift can be disregarded for infinite crystalline solids for two reasons:

(i) As discussed in the preceding section, there is no absolute zero of the potential associated with the vacuum level in infinite periodic solids.

(ii) As the extent of the system is infinite, the exchange potential does not asymptotically approach any constant value that could be associated with $c_{\text {shift }}$.

One of the quantities considered in the present paper is the fundamental band gap. According to Eq. (1) this is the $\mathrm{KS}$ gap plus the contribution from the derivative discontinuity $\Delta_{\mathrm{xc}}$. Standard (semi)local xc functionals have no derivative discontinuity, $\Delta_{\mathrm{xc}}=0$, while one can show that the AK13 potential for finite systems jumps discontinuously when the ensemble-averaged particle number in DFT [23] is changed across an integer value [13]. This feature is traditionally closely associated with the derivative discontinuity since the shift of the potential should be equal to $\Delta_{\mathrm{xc}}$. The shift of the AK13 potential comes entirely from $c_{\text {shift }}$, which changes discontinuously when a new orbital is occupied. This property of AK13 suggests a strong advantage over other (semi)local functionals in this respect. In Ref. [13] there is an explicit formula for the potential shift,

$$
c_{\text {shift }}^{i} \rightarrow-\frac{A_{x}^{2} Q_{x}^{2}}{2}\left(1 \pm \sqrt{1-\frac{4 \epsilon_{i}}{A_{x}^{2} Q_{x}^{2}}}\right),
$$

and the derivative discontinuity takes the form of

$$
\Delta_{\mathrm{xc}}=c_{\text {shift }}^{H+1}-c_{\text {shift }}^{H},
$$

where the eigenvalues are counted in $i, H$ is the index of the highest occupied eigenvalue, and $Q_{x}=\left(\sqrt{2} /\left(3\left(3 \pi^{2}\right)^{1 / 3}\right)\right) B_{1}$. Unfortunately, this formula requires knowledge of the correct absolute (unshifted) KS eigenvalues of the calculation. As explained above, these are not accessible in a computation of a crystalline solid.

Ongoing work is aimed at resolving the problem of calculating the potential shift for AK13 in periodic systems and of realizing the AK13 idea of a nonvanishing asymptotic constant in a different way. However, in this paper we focus on the orbital improvement and band gap obtained for the AK13 $\mathrm{KS}$ orbitals themselves. Hence, we follow a frequent practice used for band structure calculations using xOEP and hybrid functionals: We make no attempt to further widen the gap with $\Delta_{\mathrm{xc}}$, even though it is clear that this contribution exists. One should also note that it has been suggested that in $\Delta_{\mathrm{xc}}$ the exchange and correlation parts may have a tendency to cancel in a way that makes this contribution small for certain classes of systems [3,37].

\section{COMPUTATIONAL DETAILS}

\section{A. Electronic structure calculations}

We perform band structure calculations for crystalline solids using KS-DFT in the projector augmented wave (PAW) formalism [44] as implemented in the Vienna ab-initio simulation package (VASP) [45-48]. We have implemented the AK13 functional in this code and compute the electronic structure both with this functional and with the widely used generalizedgradient approximation by Perdew, Burke, and Ernzerhof (PBE) [49]. The cutoff energy for the plane wave expansion and the $k$-point meshes are chosen to converge the total energy and the eigenvalues of the occupied bands to $1 \mathrm{meV}$ (obtained self-consistently for both PBE and AK13). The experimental lattice constants and other computational parameters are given in the appendix (Table A1). Spin polarization is only taken into account for $\mathrm{NiO}$, where both PBE and AK13 predict antiferromagnetic order.

Where possible, we compare the KS band gap from our calculations to results from xOEP and the TB-mBJ potential [11] from the literature. The latter is a modification of $v_{x}^{\mathrm{BJ}}(\mathbf{r})$ with the aim to better reproduce experimental gaps.

\section{B. Optical dielectric constants}

We also investigate how the AK13 functional affects the optical dielectric constant. We first evaluate the independentparticle polarizability $\chi^{0}$, following the approach by Baroni and Resta [50] and Gajdoš et al. [51].

$\chi^{0}$ can be written as $[52,53]$

$$
\begin{aligned}
\chi_{\mathbf{G}, \mathbf{G}^{\prime}}^{0}(\mathbf{q}, \omega)= & \lim _{\eta \rightarrow 0} \frac{2}{\Omega} \sum_{i, j, \mathbf{k}} w_{\mathbf{k}} \frac{f\left(\varepsilon_{i, \mathbf{k}+\mathbf{q}}\right)-f\left(\varepsilon_{j, \mathbf{k}}\right)}{\varepsilon_{i, \mathbf{k}+\mathbf{q}}-\varepsilon_{j, \mathbf{k}}-\omega+i \eta}\left\langle\psi_{i, \mathbf{k}+\mathbf{q}}\right| \\
& \times e^{i(\mathbf{G}+\mathbf{q}) \cdot \mathbf{r}}\left|\psi_{j, \mathbf{k}}\right\rangle\left\langle\psi_{j, \mathbf{k}}\left|e^{-i\left(\mathbf{G}^{\prime}+\mathbf{q}\right) \cdot \mathbf{r}^{\prime}}\right| \psi_{i, \mathbf{k}+\mathbf{q}}\right\rangle . \quad(8)
\end{aligned}
$$

$\chi_{\mathbf{G}, \mathbf{G}^{\prime}}^{0}$ for given reciprocal lattice vectors $\mathbf{G}$ and $\mathbf{G}^{\prime}$, depends on the wave-vector $\mathbf{q}$ and the frequency $\omega$. For simplicity we drop the spin dependence of the KS single particle eigenstates and eigenenergies, $\psi_{j, \mathbf{k}}$ and $\varepsilon_{j, \mathbf{k}}$. With the Fermi occupation function $f\left(\varepsilon_{j, \mathbf{k}}\right)$ varying between 0 and 1 , the spin degeneracy is accounted for by the prefactor $2 / \Omega$, where $\Omega$ denotes the unit-cell volume. A small parameter $\eta$ is present in order to shift the poles of the function in the complex plane away from the real axis. In Eq. (8) the summation goes over all single particle states $i, j$ and over all $k$ points with weighting factor $w_{\mathbf{k}}$.

The dielectric matrix can be expressed in terms of the independent-particle polarizability $[50,54]$ as

$$
\epsilon_{\mathbf{G}, \mathbf{G}^{\prime}}(\mathbf{q}, \omega)=1-\frac{v_{\mathbf{G}, \mathbf{G}^{\prime}}(\mathbf{q}) \chi_{\mathbf{G}_{,}, \mathbf{G}^{\prime}}^{0}(\mathbf{q}, \omega)}{1-\sum_{G^{\prime \prime}} \chi_{\mathbf{G}, \mathbf{G}^{\prime \prime}}^{0}(\mathbf{q}, \omega) f_{\mathbf{G}^{\prime \prime}, \mathbf{G}^{\prime}}^{\mathrm{xc}}},
$$

where $f^{\mathrm{xc}}$ is the $\mathrm{xc}$ kernel

$$
f_{\mathbf{G}^{\prime \prime}, \mathbf{G}^{\prime}}^{\mathrm{xc}}=\frac{\delta^{2} E_{\mathrm{xc}}}{\delta n_{\mathbf{G}^{\prime \prime}} \delta n_{\mathbf{G}^{\prime}}}
$$

and $v$ is the Coulomb kernel:

$$
v_{\mathbf{G}, \mathbf{G}^{\prime}}(\mathbf{q})=\frac{4 \pi}{|\mathbf{G}+\mathbf{q}| \cdot\left|\mathbf{G}^{\prime}+\mathbf{q}\right|} .
$$

The macroscopic dielectric matrix $\epsilon_{\text {mac }}(\omega)$ is calculated as the long wavelength limit $(q \rightarrow 0)$ of the inverse dielectric matrix [50,51], i.e.,

$$
\epsilon_{\mathrm{mac}}(\omega)=\left(\lim _{q \rightarrow 0} \epsilon_{0,0}^{-1}(\mathbf{q}, \omega)\right)^{-1} .
$$


Here we consider three levels of approximation to $\epsilon_{\mathrm{mac}}$. On the most rigorous level we combine Eqs. (9) and (12), denoted hereafter as $\epsilon_{\mathrm{mac}}^{\mathrm{DFT}}$, in which the xc kernel is evaluated numerically from the exchange potential given in Ref. [13]. This approach is exact in principle within KS-DFT, i.e., it depends only on the approximations made in solving the KS equations and in $f^{\mathrm{xc}}$, respectively.

If the influence of the xc kernel in Eq. (9) is neglected (by setting $f^{\mathrm{xc}}=0$ ) we obtain the dielectric constant in the random phase approximation (RPA) $[55,56], \epsilon_{\mathrm{mac}}^{\mathrm{RPA}}$. Furthermore, if we neglect the local field (NLF), corresponding to the off-diagonal elements of $\epsilon_{\mathbf{G}, \mathbf{G}^{\prime}}^{-1}(\mathbf{q}, \omega)$, we obtain a dielectric constant

$$
\epsilon_{\mathrm{mac}}^{\mathrm{NLF}}=\lim _{\omega \rightarrow 0} \epsilon_{0,0}(\omega) .
$$

In practice, one can avoid the sum over unoccupied KS single particle states on all three levels of theory (DFT, RPA and NLF). This is achieved by employing density functional perturbation theory to evaluate the first order correction to the wave function with respect to the wave vector $\mathbf{q}$ in Eq. (8). For more details we refer to Refs. [50] and [51].

\section{BAND STRUCTURES AND GAPS}

\section{A. Results}

Table I shows the KS band gaps $E_{g}^{\mathrm{KS}}$ calculated with the PBE and AK13 functionals, and previously published values for the $\mathrm{xOEP}$ functional and the TB-mBJ potential at experimental lattice constants (Table A1). We here regard PBE as representative of other standard (semi)local functionals, since the differences in orbital description on unrelaxed structures are usually small. For all systems the AK13 functional gives a considerably larger $E_{g}^{\mathrm{KS}}$ than the PBE functional, bringing it towards better agreement with $\mathrm{xOEP}, \mathrm{TB}-\mathrm{mBJ}$, and the experimental value.

Note that $\mathrm{Ge}, \alpha-\mathrm{Sn}$ and $\mathrm{CdO}$ are predicted as metallic by PBE, whereas xOEP, TB-mBJ, and AK13 all open band gaps. For these systems the band structure and the electronic density of states are shown in Fig. 1. For Ge, the PBE functional predicts a vanishingly small gap, whereas AK13 opens a significant band gap $(0.6 \mathrm{eV})$. Similarly, PBE predicts $\alpha$-Sn to be a metal, with bands crossing at the zone center and
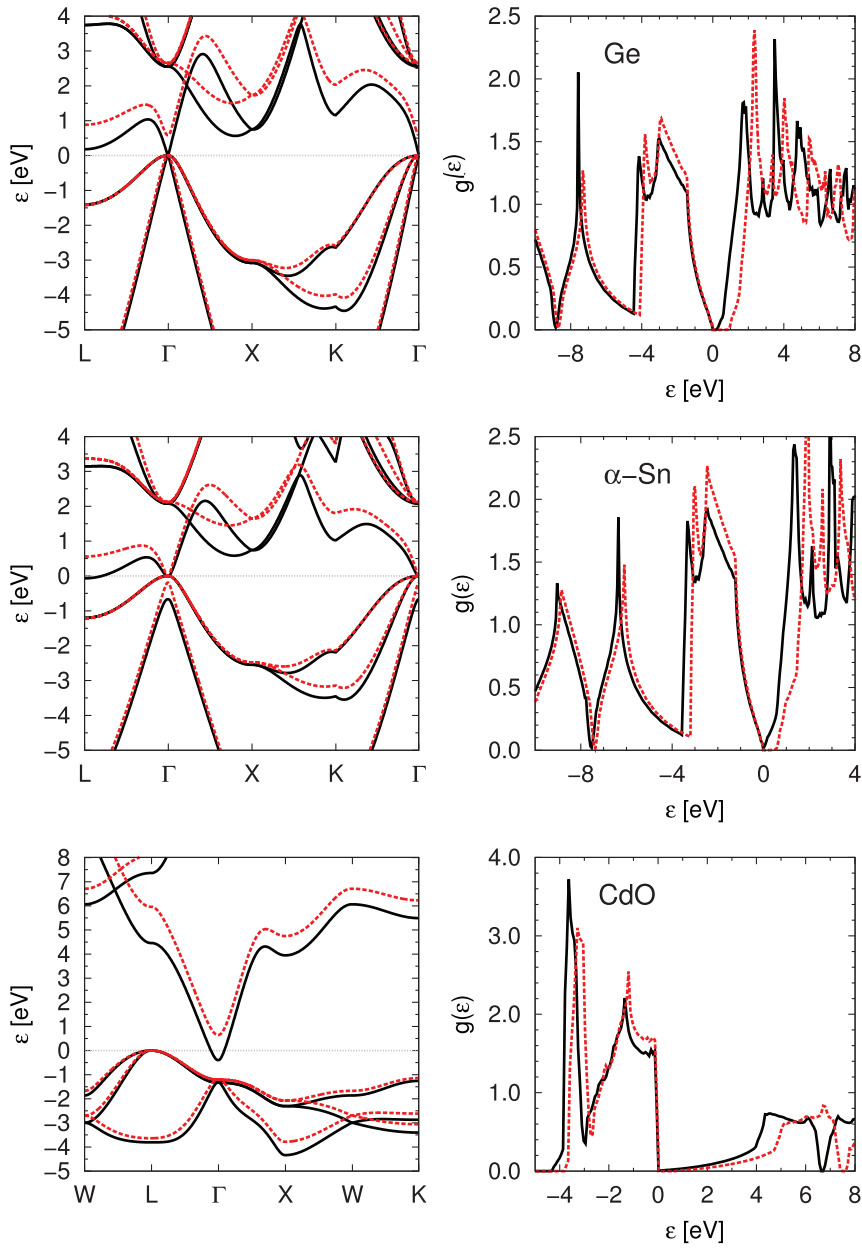

FIG. 1. (Color online) Band structure (left) and the density of states (right) for Ge (top), $\alpha$-Sn (middle), and $\mathrm{CdO}$ (bottom). PBE results are shown with a solid black line; dashed red lines represent the AK13 results. The Fermi energy is chosen as the zero of energy and is also indicated by the horizontal gray dotted line for the band structure.

close to the Brillouin zone boundary near the high symmetry point L. The AK13 functional changes the dispersion of the unoccupied bands significantly, including a shift of the valence

TABLE I. KS band gaps from the current calculations (PBE and AK13) in comparison to band gaps computed with the Tran-Blaha modified-Becke Johnson potential (TB-mBJ), exact KS exchange (xOEP), and experiments (Exp). References are given in the brackets.

\begin{tabular}{|c|c|c|c|c|c|}
\hline & PBE & AK13 & TB-mBJ & $\mathrm{xOEP}$ & Exp \\
\hline $\mathrm{C}$ (diamond) & 4.1 & 4.8 & $4.9[11]$ & $5.4[37]$ & $5.5[57]$ \\
\hline $\mathrm{Si}$ & 0.6 & 1.6 & $1.0[58]-1.2[11]$ & $1.4[59]$ & $1.1[60]$ \\
\hline $\mathrm{SiC}$ & 1.4 & 2.2 & $2.3[11]$ & $2.5[37]$ & $2.4[61]$ \\
\hline$\alpha-\mathrm{Sn}$ & -0.1 & 0.0 & & & $0.1[62]$ \\
\hline $\mathrm{Ge}$ & 0.0 & 0.6 & $0.7[58]$ & $1.0[37]$ & $0.7[63]$ \\
\hline GaAs & 0.5 & 1.4 & $1.6[64]$ & $1.5[59]$ & $1.4[65]$ \\
\hline $\mathrm{MgO}$ & 4.7 & 6.6 & $7.2[11]-8.3[58]$ & $7.8[66]$ & 7.8 [67] \\
\hline $\mathrm{NaCl}$ & 5.1 & 9.8 & & & $9.0[68]$ \\
\hline $\mathrm{CaF}_{2}$ & 7.3 & 9.8 & & & $11.8[69]$ \\
\hline $\mathrm{ZnO}$ & 0.8 & 2.0 & $2.7[70]-3.4[58]$ & & $3.4[71]$ \\
\hline $\mathrm{CdO}$ & -0.4 & 0.7 & $1.8[72]$ & & $2.3[73]$ \\
\hline $\mathrm{NiO}$ & 0.7 & 1.4 & $4.2[11]$ & $4.1[38]$ & $4.0[74]-4.2[75]$ \\
\hline
\end{tabular}


pocket near L to a conduction band, and describes the system as a semiconductor with zero band gap. For $\mathrm{CdO}$ the AK13 functional opens an indirect gap $\mathrm{L} \rightarrow \Gamma$. Opening of the gap can be seen in the density of states $\mathrm{g}(\varepsilon)$ as well (Fig. 1).

To investigate the possible influence of a correlation functional we also calculated band gaps using the AK13 exchange functional and the correlation functional of the local density approximation (LDA) for a few solids. The differences to band gaps using no correlation were minor, on the order of $0.1-0.2 \mathrm{eV}$, and similar relative shifts between the computations with and without LDA correlation were observed for eigenvalues of occupied and unoccupied bands close to the Fermi level.

\section{B. Discussion}

For the band gaps (Table I), there is a qualitative difference between $x O E P, T B-m B J$, and experimental values, on the one hand, and PBE, on the other hand. This observation corroborates a picture of something missing from standard (semi)local functionals. From the computed band gaps and the band structure in Fig. 1 it should be clear that the AK13 functional gives a distinctly different orbital description from PBE in the systems studied, with a band structure closer to what is provided by $\mathrm{xOEP}$, for example. If the difference stems from the orbital description of PBE being inaccurate due to overdelocalization, it appears that AK13 has, at least partly, addressed this deficiency. A natural consequence of mitigating the overdelocalization is the contraction in the bandwidth which can be seen in the band structures (Fig. 1, Table A2) and is even more pronounced in the density of states. The issue of describing localized states in semilocal DFT was outlined in the introduction and is discussed in more detail in Ref. [13]. It is worth noting that bandwidth contraction was previously also noted in the KS band structures obtained with the TB-mBJ potential [58].

One may at this point ask if not the better agreement with experimental results of the also arguably semilocal KS potential of TB-mBJ makes AK13 superfluous. With respect to this question we make four observations:

(i) One of the primary strengths of DFT is its rigorous theoretical framework that underpins every calculation. The $\mathrm{BJ}$ model potential is a construct that directly models the KS potential. It is an ingenious potential construction, but as such, its corresponding energy functional is not merely unknown, it does not exist [76,77], and this deficiency cannot easily be corrected [78]. Since the KS equations are derived from variational calculus of an energy equation that involves the energy xc functional, the use of BJ-type potentials has a very weak formal theoretical basis.

(ii) As mentioned above, the TB-mBJ exchange potential has been fitted to experimental band gap values, whereas AK13 was constructed without any empirical parameters.

(iii) There should be a positive contribution from $\Delta_{\mathrm{xc}}$ to the gap [Eq. (1)], therefore Kohn-Sham gaps that underestimate experimental gaps are consistent with the general theoretical expectation.

(iv) As seen from our results for AK13 exchange with LDA correlation, the correlation functional generally has a smaller impact on the band structure than exchange, but it is not completely irrelevant. The fitting done in the construction of TB-mBJ includes LDA correlation, whereas AK13 was developed to only model exchange, and so far there is no correlation functional constructed to match the features of AK13.

The more general question about the value of a KS functional that yields qualitatively improved KS gaps will be taken up again in Sec. VII-in light of the fundamental difference between $E_{g}$ and $E_{g}^{\mathrm{KS}}$.

\section{OPTICAL DIELECTRIC CONSTANTS}

\section{A. Results}

Our computed optical dielectric constants are shown in Table II. For most of the systems, there are significant but not overly clear differences in the results. If we neglect the results for $\mathrm{Ge}$ and $\mathrm{NiO}$ (discussed below), the average difference

TABLE II. The macroscopic dielectric constant computed for PBE and AK13 functionals at the three different levels of approximation discussed in Sec. III B. The different approximations are denoted by their respective abbreviations given in the superscripts: NLF (neglect of local fields), RPA (random phase approximation), and DFT (calculated on the DFT level). References for the experimental results are provided in the bracket for each value.

\begin{tabular}{|c|c|c|c|c|c|c|c|}
\hline & \multicolumn{2}{|c|}{$\epsilon_{\mathrm{mac}}^{\mathrm{NLF}}$} & \multicolumn{2}{|c|}{$\epsilon_{\mathrm{mac}}^{\mathrm{RPA}}$} & \multicolumn{2}{|c|}{$\epsilon_{\mathrm{mac}}^{\mathrm{DFT}}$} & \multirow[b]{2}{*}{ Exp. } \\
\hline & PBE & AK13 & PBE & AK13 & PBE & AK13 & \\
\hline $\mathrm{C}$ (diamond) & 6.0 & 5.6 & 5.5 & 5.2 & 5.8 & 5.8 & 5.7 [57] \\
\hline $\mathrm{Si}$ & 13.5 & 9.8 & 12.1 & 8.7 & 12.9 & 10.1 & 11.9 [57] \\
\hline $\mathrm{SiC}$ & 7.2 & 6.2 & 6.6 & 5.6 & 7.0 & 6.4 & $6.5[57]$ \\
\hline$\alpha-\mathrm{Sn}$ & 28.9 & 21.3 & 26.8 & 19.8 & 28.2 & 22.5 & $24[79,80]$ \\
\hline $\mathrm{Ge}$ & $\sim 10^{3}$ & 14.7 & $\sim 10^{3}$ & 13.4 & $\sim 10^{3}$ & 15.6 & $15.8[81]$ \\
\hline GaAs & 14.4 & 9.7 & 13.1 & 8.7 & 13.9 & 10.2 & 10.9 [82] \\
\hline $\mathrm{MgO}$ & 3.2 & 2.6 & 3.0 & 2.4 & 3.2 & 2.8 & $3.0[83]$ \\
\hline $\mathrm{NaCl}$ & 2.8 & 2.1 & 2.3 & 1.7 & 2.5 & 2.1 & $2.3[84]$ \\
\hline $\mathrm{CaF}_{2}$ & 2.4 & 2.0 & 2.3 & 1.9 & 2.3 & 2.1 & $2.0[85]$ \\
\hline $\mathrm{ZnO}$ & 5.1 & 3.6 & 4.8 & 3.4 & 5.0 & 3.7 & $3.7[86]$ \\
\hline $\mathrm{CdO}$ & 7.2 & 4.9 & 6.9 & 4.7 & 7.1 & 5.1 & 5.3 [87] \\
\hline $\mathrm{NiO}$ & 22.0 & 10.3 & 21.7 & 10.1 & 23.1 & 11.4 & $5.7[88]$ \\
\hline
\end{tabular}


between PBE and experimental values is ca. $+16 \%$ which is similar to what has previously been reported for LDA [51]. For AK13 the average difference is instead negative and of significantly reduced magnitude, ca. $-4 \%$.

To first order, one may consider the optical dielectric constant to be inversely proportional to the KS band gap; the results for AK13 can thus be regarded as a consequence of opening $E_{g}^{\mathrm{KS}}$. Nevertheless, as indicated in Sec. IV B, density functional perturbation theory is used and the actual results depend on the band structure of the system.

If we focus on the small band gap systems, the differences are clearer. For Ge, the PBE functional gives optical dielectric constants that are several orders of magnitude higher than the experimental value (ca $10^{3}$ ). For $\mathrm{NiO}$ the values differ roughly by a factor of two. There are also less striking, but still major differences for $\alpha$-Sn and $\mathrm{CdO}$.

We find that at all levels of theory $\left(\epsilon_{\mathrm{mac}}^{\mathrm{NLF}}, \epsilon_{\mathrm{mac}}^{\mathrm{RPA}}\right.$ and $\left.\epsilon_{\mathrm{mac}}^{\mathrm{DFT}}\right)$ AK13 generally gives a lower value of the optical dielectric constant than PBE. At the RPA level, both functionals give the lowest values, and when accounting for the xc kernel, i.e., at the DFT level, the dielectric constant increases. While a detailed numerical comparison between the various methods and experiments is not the focus of our paper, we note that going from the RPA to the DFT level (i.e., taking into account the xc kernel) shifts the dielectric constant of PBE further away from the experimental value, while the AK13 results are brought closer towards the experimental data. The same behavior as seen here for PBE has been reported previously for LDA [50].

To investigate the influence of correlation on the dielectric constants, we performed calculations for a few solids combining AK13 exchange and LDA correlation. Correlation changes the values, but not to an extent important for the conclusions of this paper. In our tests, correlation has the strongest influence on the dielectric constants of $\alpha$-Sn, leading to a decrease in the value by $10 \%$ for all levels of approximation.

\section{B. Discussion}

As explained in Secs. I and II, in systems where standard (semi)local functionals give an overdelocalized orbital description, we expect properly localized KS orbitals to give dielectric constants in better qualitative agreement with experimental results due to their increased similarity to the true quasiparticle electron states. Hence, our central argument is that the fact that the AK13 functional moves the dielectric constant distinctly towards the experimental result compared to $\mathrm{PBE}$ is a clear indicator of an improved orbital description. The differences between PBE and AK13 in dielectric constants (Table II) for large gap systems are not major, so we focus in the following on the systems for which PBE predicts a metallic state or a very small band gap: $\mathrm{Ge}, \alpha-\mathrm{Sn}$, and $\mathrm{CdO}$.

The large value of the dielectric constant of Ge with PBE is directly related to the zero or vanishingly small direct gap: In the limit of $\mathbf{q} \rightarrow 0$ and $\omega \rightarrow 0$, the fraction in the sum in Eq. (8) can simply be viewed as a derivative of the occupation function with respect to the KS eigenstate energy at a given wave vector $\mathbf{k}, \partial f\left(\varepsilon_{i, \mathbf{k}}\right) / \partial \varepsilon_{i, \mathbf{k}}$. In the case of Ge (see Fig. 1), the PBE valence and conduction band are very close to each other at $\Gamma$, providing a vanishing band gap, which gives a very large contribution to $\epsilon_{\mathrm{mac}}$. Opening the gap by using the AK13 functional thus reduces the values of the optical dielectric constants at all levels of theory and leads to results comparable to experiments.

For the other two solids of interest, $\alpha$-Sn and $\mathrm{CdO}$, the values of $\epsilon_{\mathrm{mac}}$ do not indicate a similar behavior. In the case of $\mathrm{CdO}$ this can be explained by the fact that the conduction band has its minimum at a $\mathbf{k}$ vector different from the wave vector of the highest occupied KS eigenstate (Fig. 1); there is thus no zero or vanishingly small direct gap.

For $\alpha$-Sn both the PBE and AK13 functional provide a vanishing direct gap at $\Gamma$. The divergence in the derivative of the occupation function is balanced by other terms in Eqs. (8) and (9), however. Applying the AK13 functional reduces the value of $\epsilon_{\text {mac }}$ relative to the PBE results and brings its value closer to the experimental data.

From Eq. (9) we see that the contribution of the $\mathrm{xc}$ functional both enters via the KS orbital shapes used in Eq. (8), and more directly via the xc kernel $f^{\mathrm{xc}}$. Nevertheless, the difference between $\mathrm{AK} 13$ and $\mathrm{PBE}$ remains roughly equal across all levels of theory, i.e., $\epsilon_{\mathrm{mac}}^{\mathrm{NLF}}, \epsilon_{\mathrm{mac}}^{\mathrm{RPA}}$, and $\epsilon_{\mathrm{mac}}^{\mathrm{DFT}}$. Since the xc kernel is only present on the DFT level, the primary difference between PBE and AK13 dielectric constants cannot only come from this term; rather, the differences originate from qualitative differences in the KS orbitals (cf. Fig. 1 for $\alpha-\mathrm{Sn}$ ).

\section{OUTLOOK AND SUMMARY}

It is a worthwhile task to consider the results that we have obtained with the AK13 functional here in the context of the derivation of the AK13 functional as reviewed in Sec. III, and the fundamental theorems of DFT that we reviewed in Secs. I and II. While AK13 yields clear improvements, there are steps in its derivation that are nonunique. Therefore, one could hope to further improve the results by extending and modifying the AK13 approach. Three lines of further development and possible modification appear naturally.

First, the AK13 functional is for exchange only. A compatible correlation functional can change the gaps. While previous experience [37] and our results using LDA correlation indicate that typical (semi)local correlation functionals change gaps by about $0.1 \mathrm{eV}$, i.e., quite moderately, correlation corrections can be larger in general. Specifically, one would expect that they affect so-called strongly correlated systems such as $\mathrm{NiO}$, where indeed the AK13 exchange functional shows the largest deviation of all solids considered here.

Second, even without introducing correlation the AK13 functional itself could be changed. The derivation of the AK13 approach [13] was guided by conditions inferred from the asymptotic behavior of the exchange potential of finite systems. Instead, one could try to optimize the AK13 functional for solids, e.g., by fitting to experimental band gaps in the spirit of the TB-mBJ potential. Third, and in a somewhat similar vein, one could also try to obtain improved energetics together with physical eigenvalues by changing the enhancement factor in Eq. (5), e.g., via changing $B_{1}$.

In this paper we deliberately pursue neither of these options. One of our reasons is that using the parameters that are present in the AK13 approach as fit parameters would lead away from the first principles character of the AK13 concept. More 
important yet is another reason: Fitting the parameters in AK13 such that its KS gaps match experimental band gaps would ignore that the fundamental gap in KS DFT is built from the $\mathrm{KS}$ gap and $\Delta_{\mathrm{xc}}$ [Eq. (1)]. Therefore, there is no fundamental reason to expect that such fitting would lead to a functional with transferable accuracy for unoccupied eigenvalues and band gaps. Indeed, it is a reoccurring experience that reliable eigenvalues and binding energies are not easily obtained from one and the same functional [89-91].

While we therefore argue that trying to obtain quantitatively accurate predictions for experimental gaps from $\mathrm{KS}$ gaps is futile, we also argue that it is nevertheless very important to have functionals that predict the electronic density of states in a qualitatively correct way. A hallmark problem of standard (semi)local functionals is that they do not properly capture localization effects. As a consequence, the ordering of the KS orbitals can be wrong, leading to a qualitatively wrong KS gap and other qualitative failures, e.g., grossly misleading predictions for charge transfer [92,93]. On the other hand, when the Slater determinant comprised of KS orbitals captures the physics of the true many-body wave function in a qualitatively correct way, then reliable understanding and insight can already be gained from the KS states. This has been demonstrated impressively, e.g., by photoemission experiments [20-22]. We have shown here that the AK13 functional can remedy some of the worst qualitative failures of typical (semi)local functionals. This is an important step forward for two reasons. The first is a conceptual one and within DFT: The KS band gap is an important contribution to the true gap, and even the exact $\Delta_{\mathrm{xc}}$ contribution will not yield correct results when added to a qualitatively wrong KS gap. The second goes beyond DFT: Today, results from DFT methods are not only valuable in and of themselves, but are also important as input to higher order methods such as the GW approach. The accuracy of such methods, specifically GW, for systems with a complex electronic structure can depend sensitively on the DFT input [34,94-96]. A functional such as AK13 that remedies the worst failures already on the DFT level may serve as a better starting point than standard (semi)local functionals.

In summary, we have compared KS band gaps and optical dielectric constants obtained with AK13 to results from established DFT approaches (PBE, xOEP, TB-mBJ) and experiments. The AK13 functional gives larger KS band gaps than a standard GGA and brings a qualitative improvement in computed macroscopic dielectric constants for some systems. In particular, for $\mathrm{Ge}, \mathrm{CdO}$, and $\alpha$-Sn the $\mathrm{AK} 13$ functional opens a band gap and thus remedies a qualitative failure of standard (semi)local functionals. As such, the AK13 band structure may serve as an improved and inexpensive starting point for higher level DFT methods [97] or beyond-DFT (GW) calculations.

\section{ACKNOWLEDGMENTS}

Computations were performed at the Leibniz Supercomputing Centre of the Bavarian Academy of Sciences and the Humanities. This work was supported by the Deutsche Forschungsgemeinschaft (DFG) under contract STE1105/8-1 to GSN and SFB840 project B1 to SK. R.A. acknowledges financial support from the Swedish Research Council (VR)
TABLE A1. Computational parameters used in the current calculations. The experimental lattice constants in $\AA$ (with the appropriate references given in the brackets) are provided together with the $k$-point meshes and energy cutoff energies for the plane-wave expansion $E^{C}$ in $\mathrm{eV}$ used here. The lattice constants are given for the conventional unit cells. Note that for $\mathrm{ZnO}$ the first value represents the length of the $a$ and $b$ axes and the latter value is for the $c$ axis. It is important to mention that the $k$-point mesh for $\mathrm{NiO}$ is given for the supercell used to generate the antiferromagnetic order. For the calculation of the dielectric constants for $\mathrm{ZnO}$ a smaller $E^{C}(1000 \mathrm{eV})$ and a coarser $k$-point mesh $(16 \times 16 \times 16)$ is used.

\begin{tabular}{lccc}
\hline \hline & $a_{0}$ & $k$ points & $E^{C}$ \\
\hline $\mathrm{C}$ (diamond) & $3.567[98]$ & $22 \times 22 \times 22$ & 1800 \\
$\mathrm{Si}$ & $5.431[99]$ & $22 \times 22 \times 22$ & 1200 \\
$\mathrm{SiC}$ & $4.358[100]$ & $22 \times 22 \times 22$ & 1600 \\
$\alpha-\mathrm{Sn}$ & $6.490[101]$ & $22 \times 22 \times 22$ & 1400 \\
$\mathrm{Ge}$ & $5.657[98]$ & $22 \times 22 \times 22$ & 1400 \\
$\mathrm{GaAs}$ & $5.654[98]$ & $22 \times 22 \times 22$ & 1000 \\
$\mathrm{MgO}$ & $4.213[102]$ & $22 \times 22 \times 22$ & 1600 \\
$\mathrm{NaCl}$ & $5.653[103]$ & $24 \times 24 \times 24$ & 1200 \\
$\mathrm{CaF}$ & $5.463[104]$ & $24 \times 24 \times 24$ & 1400 \\
$\mathrm{ZnO}$ & $3.234,5.177[105]$ & $22 \times 22 \times 16$ & 1100 \\
$\mathrm{CdO}$ & $4.696[106]$ & $24 \times 24 \times 24$ & 1100 \\
$\mathrm{NiO}$ & $4.183[107]$ & $12 \times 12 \times 12$ & 1200 \\
\hline \hline
\end{tabular}

Grant No. 621-2011-4249 and the Linnaeus Environment at Linköping on Nanoscale Functional Materials (LiLi-NFM) funded by VR.

\section{APPENDIX}

In Table A1 the computational parameters for the electronic structure calculations are summarized. In Table A 2 we present widths of the conduction and first valence band calculated with the PBE and AK13 functionals, together with results from experiments and computations using TB-mBJ [58],

TABLE A2. Valence band widths (V) and first conduction band width $\left(\mathrm{C}_{1}\right)$ from the current calculations using the PBE and AK13 functionals in $\mathrm{eV}$. TB-mBJ and experimental values are taken from the literature where available. References are provided in the bracket for each experimental value and the TB-mBJ results are from Ref. [58].

\begin{tabular}{|c|c|c|c|c|c|c|c|}
\hline & \multicolumn{2}{|c|}{ PBE } & \multicolumn{2}{|c|}{ AK13 } & \multicolumn{2}{|c|}{ TB-mBJ } & \multirow{2}{*}{$\begin{array}{c}\text { Exp. } \\
\text { V }\end{array}$} \\
\hline & V & $\mathrm{C}_{1}$ & $\mathrm{~V}$ & $\mathrm{C}_{1}$ & V & $\mathrm{C}_{1}$ & \\
\hline $\mathrm{C}$ (diamond) & 21.5 & 6.1 & 21.6 & 6.4 & & & 23.0 [108] \\
\hline $\mathrm{Si}$ & 12.0 & 3.4 & 11.6 & 3.2 & 11.7 & 3.5 & 12.5 [109] \\
\hline $\mathrm{SiC}$ & 8.5 & 5.8 & 8.1 & 5.4 & & & \\
\hline$\alpha-\mathrm{Sn}$ & 10.7 & 3.2 & 10.4 & 3.5 & & & \\
\hline $\mathrm{Ge}$ & 12.8 & 3.9 & 12.5 & 3.9 & 12.3 & 4.1 & 12.6 [109] \\
\hline GaAs & 6.8 & 3.9 & 6.5 & 3.4 & & & \\
\hline $\mathrm{MgO}$ & 4.6 & 6.8 & 4.0 & 6.8 & 3.7 & 6.1 & 4.8 [110] \\
\hline $\mathrm{NaCl}$ & 1.9 & 3.9 & 1.2 & 2.5 & & & \\
\hline $\mathrm{CaF}_{2}$ & 2.7 & 2.1 & 2.1 & 0.3 & & & \\
\hline $\mathrm{ZnO}$ & 6.2 & 7.0 & 5.9 & 6.8 & 5.4 & 6.6 & 9.0 [111] \\
\hline $\mathrm{CdO}$ & 4.3 & 7.0 & 3.8 & 6.8 & & & \\
\hline $\mathrm{NiO}$ & 7.6 & 1.2 & 7.0 & 0.6 & & & \\
\hline
\end{tabular}


where available. This follows the discussion in Ref. [58] where the localization of electronic bands (band dispersion) is considered. With the exception of Ge all the calculations show smaller band widths than the experiments. PBE yields the largest band dispersion, while the AK13 and TB-mBJ results have similar widths.
[1] W. Kohn and L. J. Sham, Phys. Rev. 140 A1133 (1965).

[2] P. Hohenberg and W. Kohn, Phys. Rev. 136, B864 (1964).

[3] S. Kümmel and L. Kronik, Rev. Mod. Phys. 80, 3 (2008).

[4] A. D. Becke, J. Chem. Phys. 98, 5648 (1993).

[5] J. Heyd, G. Scuseria, and M. Ernzerhof, J. Chem. Phys. 118, 8207 (2003).

[6] R. T. Sharp and G. K. Horton, Phys. Rev. 90, 317 (1953).

[7] J. D. Talman and W. F. Shadwick, Phys. Rev. A 14, 36 (1976).

[8] L. Hedin, Phys. Rev. 139, A796 (1965).

[9] V. I. Anisimov, F. Aryasetiawan, and A. I. Lichtenstein, J. Phys.: Condens. Matter 9, 767 (1997).

[10] A. D. Becke and E. R. Johnson, J. Chem. Phys. 124, 221101 (2006).

[11] F. Tran and P. Blaha, Phys. Rev. Lett. 102, 226401 (2009).

[12] E. Räsänen, S. Pittalis, and C. R. Proetto, J. Chem. Phys. 132, 044112 (2010).

[13] R. Armiento and S. Kümmel, Phys. Rev. Lett. 111, 036402 (2013).

[14] T. F. T. Cerqueira, M. J. T. Oliveira, and M. A. L. Marques, J. Chem. Theory Comput. 10, 5625 (2014).

[15] C.-O. Almbladh and U. von Barth, Phys. Rev. B 31, 3231 (1985).

[16] R. G. Parr and W. Yang, Density Functional Theory of Atoms and Molecules (Oxford Science Publications, Oxford, 1989).

[17] O. Gunnarsson and K. Schönhammer, Phys. Rev. Lett. 56, 1968 (1986).

[18] Z. H. Levine and D. C. Allan, Phys. Rev. Lett. 63, 1719 (1989).

[19] D. P. Chong, O. V. Gritsenko, and E. J. Baerends, J. Chem. Phys. 116, 1760 (2002).

[20] P. Puschnig, S. Berkebile, A. J. Fleming, G. Koller, K. Emtsev, T. Seyller, J. D. Riley, C. Ambrosch-Draxl, F. P. Netzer, and M. G. Ramsey, Science 326, 702 (2009).

[21] M. Dauth, T. Körzdörfer, S. Kümmel, J. Ziroff, M. Wiessner, A. Schöll, F. Reinert, M. Arita, and K. Shimada, Phys. Rev. Lett. 107, 193002 (2011).

[22] D. Lüftner, T. Ules, E.-M. Reinisch, G. Koller, S. Soubatch, F. S. Tautz, M. G. Ramsey, and P. Puschnig, Proc. Natl. Acad. Sci. USA 111, 605 (2014).

[23] J. P. Perdew, R. G. Parr, M. Levy, and J. L. Balduz, Phys. Rev. Lett. 49, 1691 (1982).

[24] J. P. Perdew and M. Levy, Phys. Rev. Lett. 51, 1884 (1983).

[25] L. J. Sham and M. Schlüter, Phys. Rev. Lett. 51, 1888 (1983).

[26] R. W. Godby, M. Schlüter, and L. J. Sham, Phys. Rev. Lett. 56, 2415 (1986).

[27] L. Kronik, T. Stein, S. Rafaely-Abramson, and R. Baer, J. Chem. Theory Comput. 8, 1515 (2012).

[28] M. S. Hybertsen and S. G. Louie, Phys. Rev. B 34, 5390 (1986).

[29] X. Zhu and S. G. Louie, Phys. Rev. B 43, 14142 (1991).

[30] E. J. Baerends, O. V. Gritsenko, and R. van Meer, Phys. Chem. Chem. Phys. 15, 16408 (2013).

[31] A. Görling, Phys. Rev. A 54, 3912 (1996).

[32] C. Filippi, C. J. Umrigar, and X. Gonze, J. Chem. Phys. 107, 9994 (1997).
[33] G. Onida, L. Reining, and A. Rubio, Rev. Mod. Phys. 74, 601 (2002).

[34] P. Rinke, A. Qteish, J. Neugebauer, C. Freysoldt, and M. Scheffler, New J. Phys. 7, 126 (2005).

[35] P. Duffy, D. P. Chong, M. E. Casida, and D. R. Salahub, Phys. Rev. A 50, 4707 (1994).

[36] M. Städele, J. A. Majewski, P. Vogl, and A. Görling, Phys. Rev. Lett. 79, 2089 (1997).

[37] M. Städele, M. Moukara, J. A. Majewski, P. Vogl, and A. Görling, Phys. Rev. B 59, 10031 (1999).

[38] E. Engel and R. N. Schmid, Phys. Rev. Lett. 103, 036404 (2009).

[39] M. Betzinger, C. Friedrich, A. Görling, and S. Blügel, Phys. Rev. B 85, 245124 (2012).

[40] T. M. Henderson, J. Paier, and G. E. Scuseria, Phys. Status Solidi B 248, 767 (2011).

[41] J. P. Perdew and A. Zunger, Phys. Rev. B 23, 5048 (1981).

[42] T. Körzdörfer, S. Kümmel, N. Marom, and L. Kronik, Phys. Rev. B 79, 201205(R) (2009).

[43] T. Körzdörfer and S. Kümmel, Phys. Rev. B 82, 155206 (2010).

[44] D. Vanderbilt, Phys. Rev. B 41, 7892 (1990).

[45] G. Kresse and J. Furthmüller, Phys. Rev. B 54, 11169 (1996).

[46] G. Kresse and J. Furthmüller, Comput. Mater. Sci. 6, 15 (1996).

[47] G. Kresse and J. Hafner, Phys. Rev. B 49, 14251 (1994).

[48] G. Kresse and J. Hafner, Phys. Rev. B 47, 558 (1993).

[49] J. P. Perdew, K. Burke, and M. Ernzerhof, Phys. Rev. Lett. 77, 3865 (1996).

[50] S. Baroni and R. Resta, Phys. Rev. B 33, 7017 (1986).

[51] M. Gajdoš, K. Hummer, G. Kresse, J. Furthmüller, and F. Bechstedt, Phys. Rev. B 73, 045112 (2006).

[52] S. L. Adler, Phys. Rev. 126, 413 (1962).

[53] N. Wiser, Phys. Rev. 129, 62 (1963).

[54] P. E. Van Camp, V. E. Van Doren, and J. T. Devreese, Phys. Rev. B 24, 1096 (1981).

[55] H. Ehrenreich and M. H. Cohen, Phys. Rev. 115, 786 (1959).

[56] P. Nozières and D. Pines, Phys. Rev. 109, 762 (1958).

[57] P. Y. Yu and M. Cardona, Fundamentals of Semiconductions (Springer-Verlag, Berlin, 2001).

[58] D. Waroquiers, A. Lherbier, A. Miglio, M. Stankovski, S. Poncé, M. J. T. Oliveira, M. Giantomassi, G. M. Rignanese, and X. Gonze, Phys. Rev. B 87, 075121 (2013).

[59] A. Fleszar, Phys. Rev. B 64, 245204 (2001).

[60] R. S. Muller and R. I. Kamins, Device Electronics for Integrated Circuits, 2nd ed (Wiley, New York, 1986).

[61] R. G. Humphreys, D. Bimberg, and W. J. Choyke, Solid State Commun. 39, 163 (1981).

[62] A. W. Ewald and E. E. Kohnke, Phys. Rev. 97, 607 (1955).

[63] O. Madelung, ed., Semiconductors: Intrinsic Properties of Group IV Elements and III-V, II-VI, and I-VII Compounds, Vol. 22a (Springer, Berlin, 1985).

[64] H. Jiang, J. Chem. Phys. 138, 134115 (2013). 
[65] J. L. Shay, Phys. Rev. B 2, 803 (1970).

[66] T. Kotani, Phys. Rev. B 50, 14816 (1994).

[67] R. C. Whited, C. J. Flatten, and W. C. Walker, Solid State Commun. 13, 1903 (1973).

[68] D. M. Roessler and W. C. Walker, Phys. Rev. 166, 599 (1968).

[69] T. Tsujibayashi, K. Toyoda, S. Sakuragi, M. Kamada, and M. Itoh, Appl. Phys. Lett. 80, 2883 (2002).

[70] D. Koller, F. Tran, and P. Blaha, Phys. Rev. B 83, 195134 (2011).

[71] D. C. Reynolds, D. C. Look, B. Jogai, C. W. Litton, G. Cantwell, and W. C. Harsch, Phys. Rev. B 60, 2340 (1999).

[72] H. Dixit, R. Saniz, S. Cottenier, D. Lamoen, and B. Partoens, J. Phys.: Condens. Matter 24, 205503 (2012).

[73] F. P. Koffyberg, Phys. Rev. B 13, 4470 (1976).

[74] S. Hüfner, Adv. Phys. 43, 183 (1994).

[75] S. L. Dudarev, G. A. Botton, S. Y. Savrasov, C. J. Humphreys, and A. P. Sutton, Phys. Rev. B 57, 1505 (1998).

[76] A. Karolewski, R. Armiento, and S. Kümmel, J. Chem. Theory Comput. 5, 712 (2009).

[77] A. P. Gaiduk and V. N. Staroverov, J. Chem. Phys. 131, 044107 (2009).

[78] A. Karolewski, R. Armiento, and S. Kümmel, Phys. Rev. A 88, 052519 (2013)

[79] C. F. Lavine and A. W. Ewald, J. Phys. Chem. Solids 32, 1121 (1971).

[80] R. E. Lindquist and A. W. Ewald, Phys. Rev. 135, A191 (1964).

[81] R. F. Potter, Handbook of Optical Constants of Solids (Academic, New York, 1985).

[82] K. G. Hambleton, C. Hilsum, and B. R. Holeman, Proc. Phys. Soc. (UK) 77, 1147 (1961).

[83] A. E. Huges and B. Henderson, Point defects in solids (Plenum Press, New York, 1972).

[84] J. R. Hardy and A. M. Karo, Phys. Rev. B 26, 3327 (1982).

[85] T. Passerat de Silans, I. Maurin, P. Chaves de Souza Sugundo, S. Saltiel, M. P. Gorza, M. Ducloy, D. Bloch, D. de Sousa Meneses, and P. Echegut, J. Phys.: Condens. Matter 21, 255902 (2009).

[86] H. Yoshikawa and A. Sadao, Jpn. J. Appl. Phys. 36, 6237 (1997).

[87] H. Finkenrath, H. Köhler, and M. Lochmann, Z. Angew. Phys. 21, 512 (1966).
[88] W. Reichardt, V. Wagner, and W. Kress, J. Phys. C 8, 3955 (1975).

[89] E. Johnson, private communication (2014).

[90] P. Verma and R. J. Bartlett, J. Chem. Phys. 140, 18A534 (2014).

[91] T. Schmidt, E. Kraisler, A. Makmal, L. Kronik, and S. Kümmel, J. Chem. Phys. 140, 18A510 (2014).

[92] S.-H. Ke, H. U. Baranger, and W. Yang, J. Chem. Phys. 126, 201102 (2007).

[93] D. Hofmann and S. Kümmel, Phys. Rev. B 86, 201109(R) (2012).

[94] N. Marom, X. Ren, J. E. Moussa, J. R. Chelikowsky, and L. Kronik, Phys. Rev. B 84, 195143 (2011).

[95] T. Körzdörfer and N. Marom, Phys. Rev. B 86, 041110 (2012).

[96] V. Atalla, M. Yoon, F. Caruso, P. Rinke, and M. Scheffler, Phys. Rev. B 88, 165122 (2013).

[97] J. Klimeš and G. Kresse, J. Chem. Phys. 140, 054516 (2014).

[98] R. W. G. Wyckoff, Crystal Structures, 2nd ed (New York, Interscience Publishers, 1963).

[99] D. Toebbens, N. Stuesser, K. Knorr, H. Mayer, and G. Lampert, J. Appl. Phys. 45, 1456 (1974).

[100] Z. Li and C. Bradt, J. Mater. Sci. 21, 4366 (1986).

[101] J. Thewlis and A. Davey, Nature (London) 174, 1011 (1954).

[102] J. Zhang, Phys. Chem. Miner. 27, 145 (2000).

[103] D. Walker, P. K. Verma, L. M. D. Cranswick, R. L. Jones, S. M Clark, and S. Buhre, American Mineralogist 89, 204 (2004).

[104] S. Speziale and T. S. Duffy, Phys. Chem. Miner. 29, 465 (2002).

[105] H. Sowa and H. Ahsbahs, J. Appl. Cryst. 39, 169 (2006).

[106] J. Zhang, Phys. Chem. Miner. 26, 644 (1999).

[107] A. Leineweber, H. Jacobs, and S. Hull, Inorg. Chem. 40, 5818 (2001).

[108] I. Jiménez, L. J. Terminello, D. G. J. Sutherland, J. A. Carlisle, E. L. Shirley, and F. J. Himpsel, Phys. Rev. B 56, 7215 (1997).

[109] K. H. Hellwege and A. M. Hellwege, eds., Landolt-Börnstein, Zahlenwerte und Funktionen aus Naturwissenschaften und Technik (Springer Verlag, New York, 1983).

[110] L. H. Tjeng, A. R. Vos, and G. A. Sawatzky, Surf. Sci. 235, 269 (1990).

[111] U. Özgür, Y. I. Alivov, C. Liu, A. Teke, M. A. Reshchikov, S. Doan, V. Avrutin, S.-J. Cho, and H. Morkoc, J. Appl. Phys. 98, 041301 (2005). 